\title{
Evidence for vortex staircases in the whole angular range due to competing correlated pinning mechanisms
}

\author{
A. Silhanek, L. Civale, S. Candia, and G. Nieva \\ Comisión Nacional de Energía Atómica-Centro Atómico Bariloche and Instituto Balseiro, 8400 Bariloche, Argentina \\ G. Pasquini and H. Lanza \\ Comisión Nacional de Energía Atómica-Departamento de Física, Avenida del Libertador 8250, 1429, Buenos Aires, Argentina
}

(Received 29 December 1998)

\begin{abstract}
We present measurements of the angular dependence of the irreversible magnetization of $\mathrm{YBa}_{2} \mathrm{Cu}_{3} \mathrm{O}_{7}$ single crystals with columnar defects inclined with respect to the $c$ axis. At high fields a sharp maximum centered at the tracks direction is observed. At low fields we identify a lock-in phase characterized by an angleindependent pinning strength and observe an angular shift of the peak towards the $c$ axis that originates in the misalignment between vortices and applied field in anisotropic materials. The interplay among columnar defects, twins, and intrinsic pinning by the $a b$ planes generates a variety of staircase structures. We show that correlated pinning dominates for all orientations of the applied field. [S0163-1829(99)09417-5]
\end{abstract}

A difficult aspect of the study of vortex dynamics in hightemperature superconductors in the presence of correlated disorder is the determination of flux structures for applied fields tilted with respect to the pinning potential. As threedimensional vortex configurations cannot be directly observed, our knowledge is mostly based on the analysis of the angular dependence of magnetization, susceptibility, or transport data. ${ }^{1-11}$

According to theoretical models, ${ }^{12,13}$ when the angle between the applied field $\mathbf{H}$ and the defects is smaller than the lock-in angle $\varphi_{L}$ vortices remain locked into the defects thus producing a transverse Meissner effect. For tilt angles larger than $\varphi_{L}$ and smaller than a trapping angle $\varphi_{T}$, vortices form staircases with segments pinned into different defects and connected by unpinned or weakly pinned kinks. Beyond $\varphi_{T}$, vortices will be straight and take the direction of the applied field, thus being unaffected by the correlated nature of the pinning. In principle, this picture should apply with minor differences to twins, columnar defects, and intrinsic pinning. 13

Many experiments have confirmed the directional pinning due to columnar defects, twins, and $\mathrm{Cu}-\mathrm{O}$ planes. ${ }^{1-11}$ Evidence for a locked-in phase arises from the observation of the transverse Meissner effect, ${ }^{10}$ but a quantitative determination of $\varphi_{L}(H, T)$ for columnar defects had not been done until now. The introduction of columnar defects inclined with respect to the crystallographic axis has been used $^{1,6-8,10,11}$ to discriminate their pinning effects from those due to twin boundaries, and from anisotropy effects. However, the vortex staircase configurations resulting from the combined effect of the various correlated structures had not yet been explored in detail.

In this work we report studies of the vortex pinning in $\mathrm{YBa}_{2} \mathrm{Cu}_{3} \mathrm{O}_{7}$ crystals with inclined columnar defects, for the whole range of field orientations. This allows us to determine the misalignment between the applied and internal fields due to anisotropy, as well as to identify the angular range of influence of each correlated pinning structure. We present the first determination of the lock-in angle of tracks using irreversible magnetization.
The crystal used in this study was grown by the self-flux method, ${ }^{14}$ and has dimensions $\sim 200 \times 600 \times 8.5 \mu \mathrm{m}^{3}$. Columnar defects at an angle $\Theta_{D} \approx 32^{\circ}$ from the $c$ axis and a density corresponding to a matching field $B_{\Phi}=3 \mathrm{~T}$ were introduced by irradiation with $315 \mathrm{MeV} \mathrm{Au}^{23+}$ ions at the Tandar accelerator (Buenos Aires, Argentina).

dc magnetization $\mathbf{M}$ was measured in a Quantum Design superconducting quantum interference device magnetometer with two sets of pickup coils, and both the longitudinal $\left(M_{l}\right.$, parallel to $\mathbf{H})$ and transverse $\left(M_{t}\right.$, perpendicular to $\left.\mathbf{H}\right)$ components were recorded. The sample could be rotated in situ around an axis perpendicular to $\mathbf{H}$ using a homemade device. ${ }^{15}$ The angle $\Theta$ between the normal to the crystal (that coincides with the $c$ axis) and $\mathbf{H}$ was determined from the angular dependence of the response in the Meissner state. The details of this procedure, that give us an absolute accuracy $\sim 1^{\circ}$, and relative variations between adjacent angles better than $0.2^{\circ}$, are described elsewhere. ${ }^{15}$ Careful alignment of the rotation axis with the normal to the plane of irradiation ensures that the condition $\mathbf{H} \|$ tracks can be achieved within $\sim 1^{\circ}$.

Isothermal magnetization loops $M_{l}(H)$ and $M_{t}(H)$ were recorded at fixed $\Theta$. The sample was then rotated, warmed up above $T_{c}$, and cooled down in zero field to start a new run. In this way, the initial Meissner response was recorded for each angle. We use the widths of the hysteresis $\Delta M_{l}(H)$ and $\Delta M_{t}(H)$ to calculate the modulus $M_{i}=\frac{1}{2} \mathrm{~A} \overline{\mathbf{l} M_{l}^{2}+\Delta M_{t}^{2}}$ and direction of the irreversible magnetization vector $\mathbf{M}_{i}$. It is known that in thin samples $\mathbf{M}_{i}$ is normal to the surface due to geometrical constrains, ${ }^{15,16}$ except above a critical angle $\sim 87^{\circ}$ for the geometry of our crystal. We have confirmed that $\mathbf{M}_{i} \| c$ within $1^{\circ}$, for all $\Theta<85^{\circ}$.

From now on we analyze the modulus $M_{i}$ as a function of $T, H$, and $\Theta$. Figure 1 shows $M_{i}$ versus $\Theta$ at two temperatures. For clarity, only a few values of $H$ are shown. According to the critical state Bean model, $M_{i}$ is proportional to the screening current density $J$ (which is lower than the critical current $J_{c}$ due to thermal relaxation). The geometrical factor 


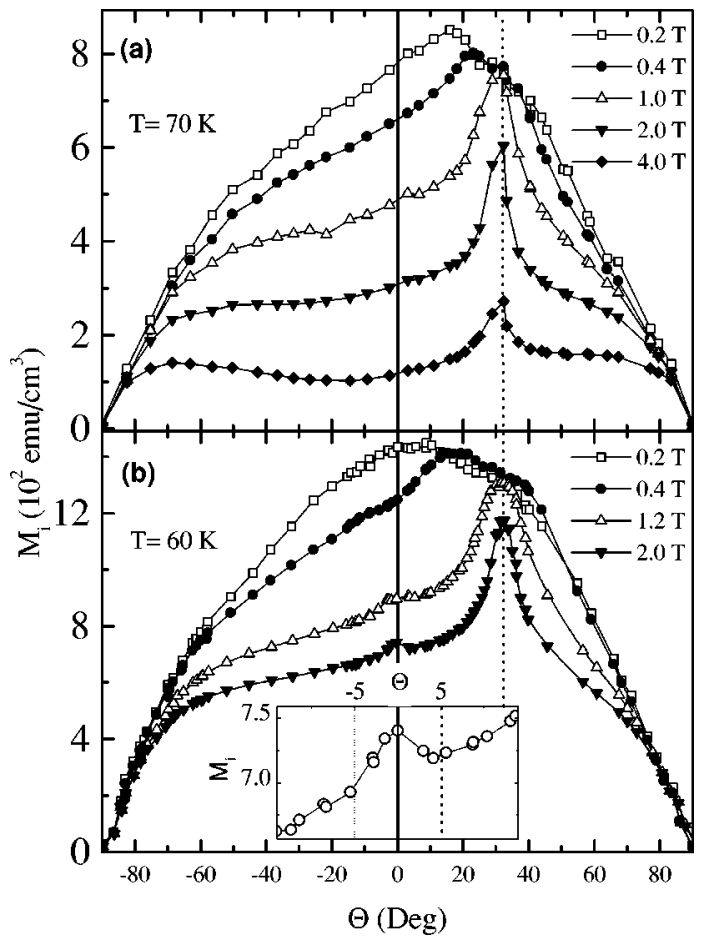

FIG. 1. Widths of the hysteresis loops $\mathbf{M}_{i}(H)$ as a function of the applied field angle $\Theta$ for several fields, at temperatures (a) $T$ $=70 \mathrm{~K}$ and (b) $T=60 \mathrm{~K}$. The inset shows a blowup of the $H$ $=2 \mathrm{~T}$ data near the $c$ axis for $T=60 \mathrm{~K}$. The units in the inset are the same of those of the main figure.

between $M_{i}$ and $J$ depends on $\Theta$, but it is almost constant for $\Theta$ lower than the critical angle. Thus the vertical axis in Fig. 1 is directly proportional to $J$ over almost all the angular range.

The most obvious feature of Fig. 1 is the asymmetry with respect to the $c$ axis, which is due to the uniaxial pinning of the inclined tracks. At high fields $(H \geqslant 1 \mathrm{~T})$ we observe a large peak in the direction of the tracks $\Theta_{D} \approx 32^{\circ}$. For $H$ $<1 \mathrm{~T}$ the peak becomes broader and progressively shifts away from the tracks in the direction of the $c$ axis as $H$ decreases. The shift decreases with increasing $T$ as shown in Fig. 2, where the angle $\Theta_{\max }$ of the maximum in $M_{i}$ is plot-

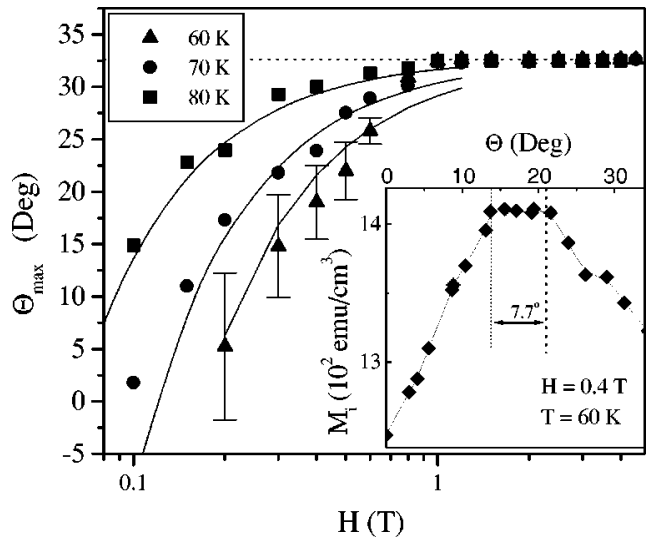

FIG. 2. Angle $\Theta_{\max }$ of the maximum in $\mathbf{M}_{i}(H)$ as a function of $H$ for three temperatures. The solid lines are fits to Eq. (1) (see text). Bars mark the width of the plateau. Inset: $\mathbf{M}_{i}(H)$ versus $\Theta_{\max }$ in the region of the plateau for $H=0.4 \mathrm{~T}$ and $T=60 \mathrm{~K}$. ted as a function of $H$ for three temperatures. The inset of Fig. 2 shows a blowup of the data of Fig. 1 for $H=0.4 \mathrm{~T}$ and $T=60 \mathrm{~K}$. This curve exhibits the second main characteristic of the low-field results, namely the existence of a plateau in $M_{i}(\Theta)$ (We define $\Theta_{\max }$ as the center of the plateau).

We first discuss the origin of the shift. Maximum pinning is expected to occur when the tracks are aligned with the direction that the vortices would have in the absence of pinning. For an anisotropic material, such direction does not coincide with $\mathbf{H}$. If $\Theta_{B}$ is the angle between the equilibrium induction field $\mathbf{B}$ (which represents the vortex direction) and the $c$ axis, minimization of the free energy for $H_{c 1}^{c} \ll H$ $\ll H_{c 2}^{c}$ gives ${ }^{13}$

$$
\sin \left(\Theta_{B}-\Theta\right) \approx \frac{H_{c 1}^{c}\left(1-\varepsilon^{2}\right)}{2 H \ln \kappa} \frac{\sin \Theta_{B} \cos \Theta_{B}}{\varepsilon\left(\Theta_{B}\right)} \ln \left(\frac{H_{c 2}\left(\Theta_{B}\right)}{B}\right),
$$

where $H_{c 2}\left(\Theta_{B}\right)=H_{c 2}^{c} / \varepsilon\left(\Theta_{B}\right)$. Here $H_{c 1}^{c}$ and $H_{c 2}^{c}$ are the lower and upper $c$ axis critical fields, $\varepsilon$ is the anisotropy and $\varepsilon(\theta)=\left(\cos ^{2} \theta+\varepsilon^{2} \sin ^{2} \theta\right)^{1 / 2}$. For $\varepsilon<1$ vortices tilt towards the $a b$ plane. When $\Theta=\Theta_{D}$ we have $\Theta_{B}>\Theta_{D}$ and the optimum pinning situation is not satisfied. Instead, maximum $M_{i}$ occurs at the vortex-track alignment condition $\Theta_{B}=\Theta_{D}$. This corresponds to an applied field angle $\Theta_{\max }<\Theta_{D}$ that can be calculated from Eq. 1 by setting $\Theta_{B}=\Theta_{D} \approx 32^{\circ}$. (Within this picture the peak cannot occur at $\Theta<0$, thus the negative values of $\Theta_{\max }$ given by Eq. 1 at low $H$ are unphysical, and $\Theta_{\max }$ must approach zero as $H \rightarrow 0$.)

The solid lines in Fig. 2 are fits to Eq. 1 with fixed parameters ${ }^{13} \varepsilon=1 / 7$ and $H_{c 2}^{c}(T)=1.6 \mathrm{~T} / \mathrm{K} \times\left(T_{c}-T\right)$ (the fits are not very sensitive to any of them). Using $H_{c 1}^{c}(T) / 2 \ln \kappa=\Phi_{0} / 8 \pi \lambda_{a b}^{2}(T) \quad$ and $\quad \lambda_{a b}^{2}(T) \approx \lambda_{a b}^{2}(0)(1$ $\left.-T / T_{c}\right)^{-1}$, we obtain a good fit to the data as a function of field and temperature by setting only one free parameter, $\lambda_{a b}(0) \approx 500 \AA$. Although this value is significantly smaller than the accepted value ${ }^{13}(\sim 1400 \AA)$, we note that this is a very simplified model, where additional effects such as demagnetizing factors, geometrical barriers, or deviations from Ginzburg-Landau theory are not included. We nevertheless consider that it captures the basic physics. We note that Zhukov et al. ${ }^{10}$ have reported lock-in angles for twin boundaries in $\mathrm{YBa}_{2} \mathrm{Cu}_{3} \mathrm{O}_{7}$ crystals that imply an $H_{c 1}^{c}$ about five times larger than the usual values, a result suggestively similar to our case. The quantitative aspects of these effects clearly require further investigation.

We now return to the plateau seen in the inset of Fig. 2. The constancy of $M_{i}(\Theta)$ indicates that the pinning energy remains constant and equal to the value at the alignment condition $\Theta_{B}=\Theta_{D}$. This behavior is a fingerprint of the lock-in phase. ${ }^{12}$ To determine the range of the plateau with good accuracy $\left(\sim 1^{\circ}\right)$, we have measured about one loop per degree at $T=60 \mathrm{~K}$ for $10^{\circ}<\Theta<40^{\circ}$. The extension of the plateau in the $H-\Theta$ plane at $60 \mathrm{~K}$ is shown as bars in Fig. 2. Its width decreases approximately as $H^{-1}$, as expected ${ }^{12}$ for $\varphi_{L}$, and for $H>1 \mathrm{~T}$ it becomes undetectable with our resolution. The decrease of $M_{i}$ at the edges of the plateau is sharp, a result consistent with the appearance of kinks, which not only reduce $J_{c}$ but also produce a faster relaxation.

When $\left|\Theta_{B}-\Theta_{D}\right|>\varphi_{L}$ vortices form staircases. Two questions arise here. First, which is the direction of the kinks that 


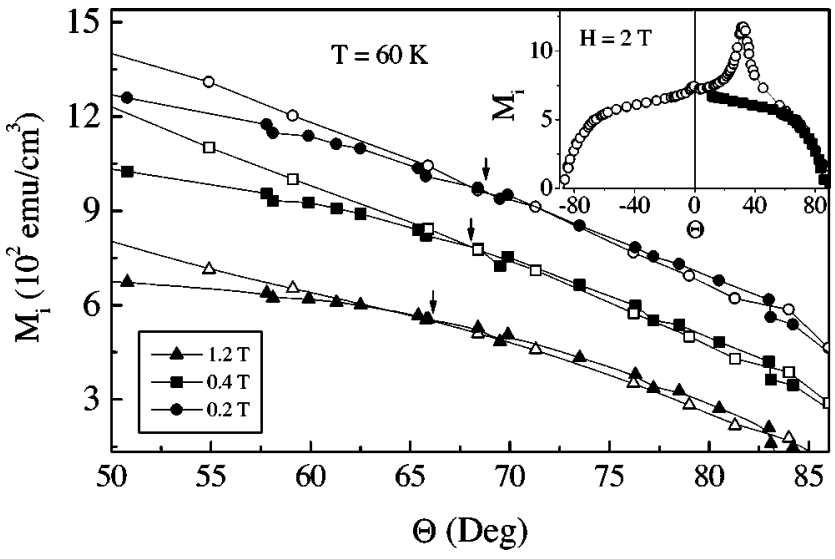

FIG. 3. Angular dependence of the irreversible magnetization $\mathbf{M}_{i}(H)$ for three fields at $T=60 \mathrm{~K}$. Open symbols: data for $\Theta>0$. Solid symbols: data for $\Theta<0$, reflected with respect to the $c$ axis. Some of the curves were displaced verically for clarity. The arrows indicate the angle $\Theta_{\text {sym }}$ beyond which the behavior is symmetric with respect to the $c$ axis. The procedure of reflection of the data is sketched in the inset.

connect the pinned portions of the vortices? Second, do we observe evidence for a trapping angle $\varphi_{T}$ ?

For $\Theta>\Theta_{\max }$, there is a wide angular range in Fig. 1 in which $M_{i}(+\Theta)>M_{i}(-\Theta)$ for all $H$, i.e., pinning is stronger when $H$ is closer to the tracks than in the crystallographically equivalent configuration in the opposite side. This asymmetry demonstrates that at the angle $+\Theta$ vortices form staircases, with segments trapped in the tracks. For $\Theta<\Theta_{\max }$ we again observe asymmetry, $M_{i}(\Theta)$ crosses $\Theta=0$ with positive slope, indicating that pinning decreases as $H$ is tilted away from the tracks. We can conclude that staircases extend at least beyond the $c$ axis into the $\Theta<0$ region.

The angle $\theta_{k}$ between the kinks and the $c$ axis can be calculated by minimization of the free energy ${ }^{13}$ For simplicity, let's consider the case $H \gg H_{c 1}^{c}$, where $\Theta_{B}=\Theta$ and the problem reduces to calculate the energy of one single vortex, as the other terms in the free-energy density are the same for all configurations. ${ }^{8,11}$ If $L_{p}$ is the length of a pinned segment, and $L_{k}$ the length of the kink (see sketch in Fig. 4), the line energy is $E \propto L_{p} \epsilon_{p}\left(\Theta_{D}\right)+L_{k} \epsilon_{f}\left(\theta_{k}\right)$, where $\epsilon_{f}\left(\theta_{k}\right)$ $\approx \varepsilon_{0} \varepsilon\left(\theta_{k}\right)[\ln \kappa+0.5]$ and $\epsilon_{p}\left(\Theta_{D}\right) \approx \varepsilon_{0} \varepsilon\left(\Theta_{D}\right)\left[\ln \kappa+\alpha_{t}\right]$ are the line energy for free and pinned vortices, respectively, $\varepsilon_{0}$ is the vortex energy scale and $\alpha_{t}<0.5$ parametrizes the core pinning energy due to the tracks (smaller $\alpha_{t}$ implies stronger pinning). Minimizing $E$ with respect to $\theta_{k}$ we obtain the two kink orientations, $\theta_{k}^{-}$for $\Theta<\Theta_{D}$ and $\theta_{k}^{+}$for $\Theta>\Theta_{D}$.

As the tracks are inclined, $\left|\theta_{k}^{-}\right|$and $\left|\theta_{k}^{+}\right|$are different. However, those angles are independent of $\Theta$. As $\left|\Theta-\Theta_{D}\right|$ increases, $\theta_{k}^{ \pm}$remain constant while $L_{p}$ decreases and the number of kinks increases, consequently the pinning energy lowers. This accounts for an $M_{i}$ that decreases as we move away from the tracks. In particular, for $\Theta=\theta_{k}^{ \pm}$vortices become straight $\left(L_{p}=0\right)$, thus $\varphi_{T}^{ \pm}=\left|\theta_{k}^{ \pm}-\Theta_{D}\right|$ are the trapping angles in both directions. In general $\theta_{k}^{ \pm}$must be obtained numerically, but for $\varepsilon \tan \theta_{k} \ll 1$ and $\varepsilon \tan \Theta_{D} \ll 1$ we obtain

$$
\tan \theta_{k}^{ \pm} \approx \tan \Theta_{D} \pm \frac{1}{\varepsilon} \overline{\ln \kappa+0.5} .
$$

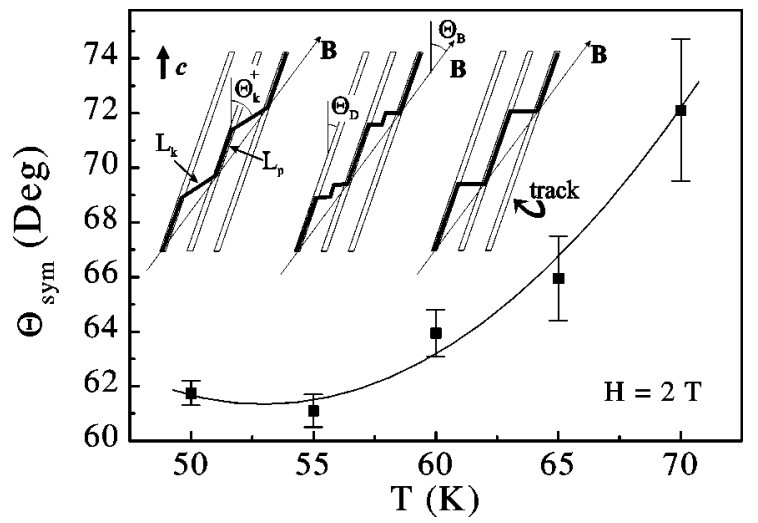

FIG. 4. Temperature dependence of $\Theta_{\text {sym }}$ (see Fig. 3). The solid line is a guide to the eye. The sketches show the possible vortex staircases for $\Theta>\Theta_{D}$.

Equation (2) adequately describes the main features of the asymmetric region in Fig. 1, and for $\Theta_{D}=0$ it coincides with the usual estimates ${ }^{12,13}$ of $\varphi_{T}$.

There is, however, an important missing ingredient in the standard description presented above, namely the existence of twins and $\mathrm{Cu}-\mathrm{O}$ layers, which are additional sources of correlated pinning. This raises the possibility that vortices may simultaneously adjust to more than one of them, forming different types of staircases.

Pinning by twin boundaries is visible in Fig. 1 as an additional peak centered at the $c$ axis for $H=2 \mathrm{~T}$ and $T$ $=60 \mathrm{~K}$. A blowup of that peak is shown in the inset. We observe this maximum for $H \geqslant 1 \mathrm{~T}$. The width of this peak, $\sim 5^{\circ}$, is in the typical range of reported trapping angles for twins. ${ }^{3-5,9,10}$ On the other hand, the fact that the peak is mounted over an inclined background implies that vortices are also trapped by the tracks. Thus vortices in this angular range contain segments both in the tracks and in the twins. These two types of segments are enough to build up the staircases for $\Theta>0$, but for $\Theta<0$ a third group of inclined kinks with $\theta_{k}<0$ must exist in order to have vortices parallel to $\mathbf{H}$.

Another fact to be considered is that the asymmetry in $M_{i}(\Theta)$ disappears as $\Theta$ approaches the $a b$ planes. This is illustrated in Fig. 3, where $M_{i}$ data for $-|\Theta|$ was reflected along the $c$ axis and superimposed to the results for $+|\Theta|$. There is a well-defined angle $\Theta_{\text {sym }}$ beyond which $M_{i}(\Theta)$ recovers the symmetry with respect to the $c$ axis. We have also found that $\Theta_{\text {sym }}$ is only weakly dependent on $H$.

One possible interpretation is that for $\Theta>\Theta_{\text {sym }}$ staircases disappear, i.e., that $\Theta_{\text {sym }}=\theta_{k}^{+}$and we are determining $\varphi_{T}^{+}$ $=\Theta_{\text {sym }}-\Theta_{D}$. However, this is inconsistent with our experimental results. Indeed, $\varphi_{T}^{+}$should decrease with $T$, and this decrease should be particularly strong above the depinning temperature ${ }^{17} T_{d p} \sim 40 \mathrm{~K}$ due to the reduction of the pinning energy by entropic smearing effects. ${ }^{12}$ This expectation is in sharp contrast with the observed increase of $\Theta_{\text {sym }}$ with temperature, which is shown in Fig. 4 for $H=2$ T. Thus the interpretation of $\Theta_{\text {sym }}$ as a measure of the trapping angle is ruled out. Moreover, if in a certain angular range vortices were not forming staircases, pinning could be described by a scalar disorder strength, then at high fields $M_{i}(\Theta)$ should follow the anisotropy scaling $\operatorname{law}^{18} M_{i}(H, \Theta)$ 
$=M_{i}[\varepsilon(\Theta) H]$. Consistently, we do not observe such scaling in any angular range.

Our alternative interpretation is that, as $\mathbf{H}$ approaches the $a b$ planes, the kinks become trapped by the intrinsic pinning. This idea has been used by Hardy et al. ${ }^{8}$ to explain that the $J_{c}$ at low $T$ in the very anisotropic $\mathrm{Bi}$ and $\mathrm{Tl}$ compounds with tracks at $\Theta_{D}=45^{\circ}$ was the same for $H$ either parallel or normal to them. Our situation is somewhat different, as we are comparing two configurations both having kinks.

We first note that, according to Eq. (2), $\theta_{k}^{ \pm}$cannot be exactly $90^{\circ}$ for finite $\varepsilon$, thus the intrinsic pinning must be incorporated into the model by assigning a lower energy to kinks in the $a b$ planes. Vortices may now form structures consisting of segments trapped in the columns connected by segments trapped in the $a b$ planes, or alternatively an inclined kink may transform into a staircase of smaller kinks connecting segments in the planes (see sketches in Fig. 4). We should now compare the energy of the new configurations with that containing kinks at angles $\theta_{k}^{ \pm}$. This is equivalent to figure out whether the kinks at $\theta_{k}^{ \pm}$lay within the trapping regime for the planes or not. The problem with this analysis is that, as $\theta_{k}^{ \pm}$are independent of $\Theta$, one of the two possibilities (either inclined or trapped kinks), will be the most favorable for all $\Theta$. Thus this picture alone cannot explain the crossover from an asymmetric to a symmetric regime in $M_{i}(\Theta)$.

The key concept to be considered in this scenario is the dispersion in the pinning energy of the tracks. The angles $\theta_{k}^{ \pm}$ depend on the pinning strength of the adjacent tracks $\left[\alpha_{t}\right.$ in Eq. (2)], thus a dispersion in $\alpha_{t}$ implies a dispersion in $\theta_{k}^{ \pm}$. As $\Theta$ increases, it becomes larger than the smaller $\theta_{k}^{ \pm}$, s (that connect the weaker defects) and the corresponding kinks disappear. The vortices involved, however, do not become straight, but remain trapped by stronger pins connected by longer kinks with larger $\theta_{k}^{ \pm}$. This process goes on as $\Theta$ grows: the weaker tracks progressively become uneffective as the "local" $\theta_{k}$ is exceeded, and the distribution of $\theta_{k}^{ \pm}$ shifts towards the $a b$ planes. When a particular kink falls within the trapping angle of the planes, a switch to the pinned-kink structure occurs. In this new picture, the gradual crossover to the symmetric regime as $|\Theta|$ increases takes place when most of the remaining kinks are pinned by the planes.

If kinks become locked, the total length of a vortex that is trapped inside columnar defects is the total length of a track, independent of $\Theta$, and the total length of the kinks is $\propto \tan \left(\left|\Theta \pm \Theta_{D}\right|\right)$ for field orientations $\pm \Theta$, respectively. As $|\Theta|$ grows, the relative difference between the line energy in both orientations decreases, an effect that is reinforced by the small line energy of the kinks in the $a b$ planes. If kinks are not locked but rather form staircases, taking into account that the trapping angle for the $a b$ planes is $\operatorname{small}^{4}\left(\sim 5^{\circ}\right)$, the same argument still applies to a good approximation. The temperature dependence of $\Theta_{\text {sym }}$ is now easily explained by a faster decrease of the pinning of the $a b$ planes with $T$ as compared to the columnar defects.

Additional evidence in support of our description comes from transport measurements in the dc-flux transformer configuration. Recent results show that, in contrast with untwinned crystals, in the liquid phase in twinned $\mathrm{YBa}_{2} \mathrm{Cu}_{3} \mathrm{O}_{7}$ crystals vortices remain correlated along the $c$ axis for all field orientations. ${ }^{19}$ This suggest that, for all angles, vortices are composed solely of segments in the twins and in the $a b$ planes.

In summary, we have shown that the combined effect of the three sources of correlated pinning must be taken into account to describe the vortex structure in samples with inclined columnar defects. We demonstrate that the lock-in phase exhibits an angle independent pinning strength, and show the decrease of the lock-in angle with field. Our results show that a variety of complex staircases are formed depending on the field orientation and strongly suggest that, at high temperatures, correlated structures dominate vortex pinning over random disorder in the whole angular range.

Work partially supported by ANPCyT, Argentina, PICT 97 No. 01120. A.S. and G.N. were partially supported by CONICET. We acknowledge useful discussions with S. Grigera, F. de la Cruz, E. Osquiguil, and D. Niebieskikwiat.
${ }^{1}$ L. Civale et al., Phys. Rev. Lett. 67, 648 (1991).

${ }^{2}$ W. K. Kwok et al., Phys. Rev. Lett. 69, 3370 (1992).

${ }^{3}$ I. V. Grigorieva, L. A. Gurevich, and L. Y. Vinninkov, Physica C 195, 327 (1992).

${ }^{4}$ W. K. Kwok et al., Phys. Rev. Lett. 64, 966 (1990); S. Fleshler et al., Phys. Rev. B 47, 14448 (1993).

${ }^{5}$ J. N. Li, Phys. Rev. B 48, 6612 (1993).

${ }^{6}$ L. Klein et al., Phys. Rev. B 47, 12349 (1993); 48, 3523 (1993).

${ }^{7}$ B. Holzapfel et al., Phys. Rev. B 48, 600 (1993).

${ }^{8}$ V. Hardy et al., Phys. Rev. B 54, 656 (1996).

${ }^{9}$ M. Oussena et al., Phys. Rev. Lett. 76, 2559 (1996).

${ }^{10}$ A. A. Zhukov et al., Phys. Rev. B 56, 3481 (1997).

${ }^{11}$ A. Herbsommer et al., Physica C 304, 112 (1998).

${ }^{12}$ D. R. Nelson and V. M. Vinokur, Phys. Rev. Lett. 68, 2398
(1992); Phys. Rev. B 48, 13060 (1993).

${ }^{13}$ G. Blatter et al., Rev. Mod. Phys. 66, 1125 (1994).

${ }^{14}$ F. de la Cruz, D. López, and G. Nieva et al., Philos. Mag. B 70, 773 (1994)

${ }^{15}$ S. Candia and L. Civale, Supercond. Sci. Technol. (to be published); D. M. Casa et al. (unpublished).

${ }^{16}$ A. A. Zhukov, G. K. Perkins, Y. V. Bugoslavsky, and A. D. Caplin, Phys. Rev. B 56, 2809 (1997).

${ }^{17}$ L. Krusin-Elbaum, L. Civale, J. R. Thompson, and C. Feild, Phys. Rev. B 53, 11744 (1996).

${ }^{18}$ G. Blatter, V. B. Geshkenbein, and A. I. Larkin, Phys. Rev. Lett. 68, 875 (1992).

${ }^{19}$ E. H. J. Morré, Ph.D. thesis, Instituto Balseiro, 1997; E. H. J. Morré et al., Phys. Lett. A 233, 130 (1997). 